\title{
Relating random telegraph signal noise in metal-oxide-semiconductor transistors to interface trap energy distribution
}

\author{
A. P. van der Wel, E. A. M. Klumperink, ${ }^{\text {a) }}$ E. Hoekstra, and B. Nauta \\ IC-Design Group, University of Twente, The Netherlands
}

(Received 27 June 2005; accepted 9 September 2005; published online 27 October 2005)

\begin{abstract}
In this work, we study random telegraph signal (RTS) noise in metal-oxide-semiconductor field effect transistors when the device is periodically and rapidly cycled between an "on" and an "off" bias state. We derive the effective RTS time constants for this case using Shockley-Read-Hall statistics applied under transient conditions. In this way, we show that the oft-observed reduction in RTS noise under such bias conditions can be explained by a nonuniform (e.g., U-shaped) distribution in energy of interface traps. (C) 2005 American Institute of Physics.
\end{abstract}

[DOI: $10.1063 / 1.2128056]$

Low-frequency (LF) noise in metal-oxide-semiconductor field effect transistors (MOSFETs) is a topic of growing concern. In newer processes with smaller devices, LF noise of MOSFETs continues to play an important role. ${ }^{1}$ LF noise is not only significant in LF circuits, but also of crucial importance in high-frequency circuits where LF noise is upconverted to dominate noise performance of many common circuit blocks such as oscillators and mixers. LF noise in MOSFETs has been studied at length: Already in 1969, Hooge showed that homogenous semiconductor samples suffer from a bulk $1 / f$ noise. Though MOSFETs probably suffer from bulk $1 / f$ noise in the same way, it has since become clear that the dominant mechanism in MOSFETs is random telegraph signal (RTS) noise caused by traps at the $\mathrm{Si}-\mathrm{SiO}_{2}$ interface. $^{2,3}$ In 1991, it was observed $^{4}$ that cycling a MOSFET from inversion to accumulation reduces its LF noise. This was later linked to the emptying of traps that generate RTS noise. ${ }^{5}$ Several authors have since used this technique to reduce LF noise in analog circuits. ${ }^{6,7}$

In this letter, we use Shockley-Read-Hall statistics applied under transient conditions to explain why RTS noise in MOSFETs decreases ${ }^{8,9}$ when the device is periodically and rapidly switched between an "on" and an "off" state. We show that the effective time constants of the RTS noise change in such a way that traps closer to the center of the band gap become the dominant contributors to the LF noise of the device. If the distribution of traps in energy is U-shaped as is often reported in literature, ${ }^{10}$ this can explain the observed reduction in LF noise.

A trap is a localized energy state in the band gap with an energy level between $E_{c}$ (conduction-band energy level) and $E_{v}$ (valence-band energy level) [Fig. 1(a)]. Traps occur at the $\mathrm{Si}-\mathrm{SiO}_{2}$ interface or in the oxide and are attributed to dangling bonds or impurities. In $n$-channel MOSFETs, traps close in energy to the conductance band edge interact with the conductance-band. A trap can capture or release an electron, which causes RTS noise in two ways: First, the captured electron takes no further part in the conduction process; this is known as the $\Delta N$ effect. Second, electron capture will make the trap more negatively charged. This modulates the position of the channel and is known as "Coulomb scattering" or as a $\Delta \mu$ effect caused by and correlated to the capture

\footnotetext{
${ }^{a)}$ Electronic mail: e.a.m.klumperink@utwente.nl
}

of an electron $(\Delta N)$. This second effect ${ }^{11}$ often causes a much larger drain current fluctuation than the simple $\Delta N$ effect.

The parameters of the RTS are its amplitude and the mean time before capture $\left(\tau_{c}\right)$ and emission $\left(\tau_{e}\right)$ of an electron. On the basis of Fermi-Dirac statistics, it can be shown that: $^{12}$

$$
\begin{aligned}
& \tau_{c}=\left[\sigma(E, x) v_{\mathrm{th}} n\right]^{-1}, \\
& \tau_{e}=\left[\sigma(E, x) v_{\mathrm{th}} N_{c} \exp (-E / k T)\right]^{-1}
\end{aligned}
$$

The capture cross section of the trap is $\sigma(E, x)$, which depends on the energy level of the trap $\left(E=E_{c}-E_{T}\right)$ and its depth in the oxide $(x) . v_{\text {th }}$ is the thermal velocity of the electrons, $n$ is the electron density in the conduction band, and $N_{c}$ is the effective density of states in the conduction band.

From Eq. (1), $\tau_{c}$ is bias dependent via the bias dependency of $n . \tau_{e}$ may also be bias dependent because if the trap is situated some distance in the oxide, $E$ depends on $V_{\mathrm{Gs}}$. This is consistent with measurements of the bias dependency of RTS time constants in $n$-channel MOSFETs. ${ }^{2,13-15}$ In all cases, $\tau_{c}$ increases with decreasing $V_{\mathrm{GS}}$, and $\tau_{e}$ decreases with decreasing $V_{\mathrm{GS}}$. Assuming that $\tau_{e}$ and $\tau_{c}$ are instantaneous functions of the bias of the device, ${ }^{15}$ the effective RTS time constants when the device is periodically switched between two bias states may now be derived.

The occupancy of a trap at any given moment is: ${ }^{12}$

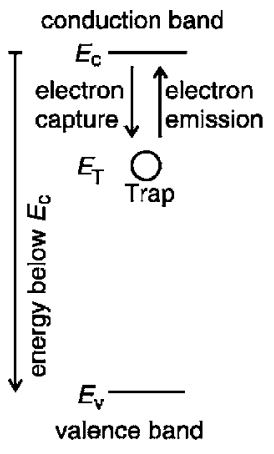

(a)

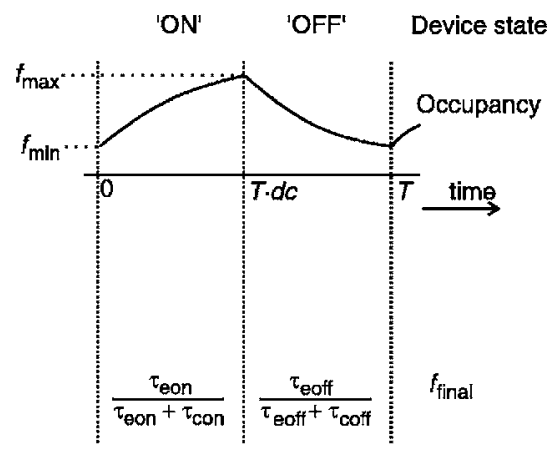

(b)
FIG. 1. (a) In n-channel MOSFETs, traps near the conduction-band edge cause RTS noise. (b) Occupancy of trap when device is cycled on and off. 


$$
f(t)=\frac{\tau_{e}}{\tau_{e}+\tau_{c}}+K \exp -\left(\frac{1}{\tau_{e}}+\frac{1}{\tau_{c}}\right) t
$$

where $K$ depends on the initial condition. We treat the case where the bias voltage alternates abruptly and periodically between two states; reference is made to Fig. 1(b). The duty cycle is $d c$; the fraction of the period in which the device is on. From $t=0 \ldots T d c$, the device is on. During this time, RTS behavior is governed by $\tau_{e, \text { on }}$ and $\tau_{c, \text { on }}$, and from $t$ $=T d c \ldots T$, the device is off, and RTS behavior is governed by $\tau_{e, \text { off }}$ and $\tau_{c, \text { off }}$. The occupancy is given by Eq. (2) at all times; the final occupancy $f_{\text {final }}$ is never reached because the switching frequency is high. Instead, the occupancy varies between $f_{\min }$ and $f_{\max }$. Since there are two states, there are a total of four time constants: $\tau_{e, \text { on }}, \tau_{e, \text { off }}, \tau_{c, \text { on }}$, and $\tau_{c, \text { off }}$.

Substituting

$$
\tau_{\text {eff }, \text { on }}=\left[\frac{1}{\tau_{e, \text { on }}}+\frac{1}{\tau_{c, \text { on }}}\right]^{-1} \text { and } \tau_{\text {eff }, \text { off }}=\left[\frac{1}{\tau_{e, \text { off }}}+\frac{1}{\tau_{c, \text { off }}}\right]^{-1}
$$

and equating $f(0)$ and $f(T)$, we may derive: ${ }^{16}$

$$
\begin{aligned}
& f_{\min }=f(T)=\frac{\frac{\tau_{e, \text { off }}}{\tau_{e, \text { off }}+\tau_{c, \text { off }}}\left(1-\exp \frac{-T(1-d c)}{\tau_{\text {eff,off }}}\right)}{1-\exp \frac{-T d c}{\tau_{\text {eff,on }}} \exp \frac{-T(1-d c)}{\tau_{\text {eff,off }}}} \\
& +\frac{\frac{\tau_{e, \mathrm{on}}}{\tau_{e, \mathrm{on}}+\tau_{c, \mathrm{on}}} \exp \frac{-T(1-d c)}{\tau_{\mathrm{eff}, \mathrm{off}}}\left(1-\exp \frac{-T d c}{\tau_{\mathrm{eff}, \mathrm{on}}}\right)}{1-\exp \frac{-T d c}{\tau_{\mathrm{eff}, \mathrm{on}}} \exp \frac{-T(1-d c)}{\tau_{\mathrm{eff}, \mathrm{off}}}},
\end{aligned}
$$

where $f_{\max }$ can be found in a similar way. If the switching frequency is made very high compared to the RTS corner frequency ( $T$ much smaller than $\tau_{e}$ and $\tau_{c}$ ), $f_{\min }$ and $f_{\max }$ converge to the same value; $f_{\text {eff }}$, and the RTS becomes stationary. This equivalent stationary RTS has time constants $\tau_{c, \text { eff }}$ and $\tau_{e, \text { eff }}$ which can be found by first deriving $f_{\text {eff }}$

$$
f_{\text {eff }}=\frac{1 /\left[\frac{d c}{\tau_{e, \text { on }}}+\frac{1-d c}{\tau_{e, \text { off }}}\right]}{1 /\left[\frac{d c}{\tau_{e, \text { on }}}+\frac{1-d c}{\tau_{e, \text { off }}}\right]+1 /\left[\frac{d c}{\tau_{c, \text { on }}}+\frac{1-d c}{\tau_{c, \text { off }}}\right]}
$$

This describes the effective occupancy of the equivalent stationary RTS and equals:

$$
f_{\text {eff }}=\frac{\tau_{e, \text { eff }}}{\tau_{e, \text { eff }}+\tau_{c, \text { eff }}}
$$

It follows that: Downloaded 18 Oct 2006 to 130.89.92.11. Redistribution subject to AIP license or copyright, see http://apl.aip.org/apl/copyright.jsp

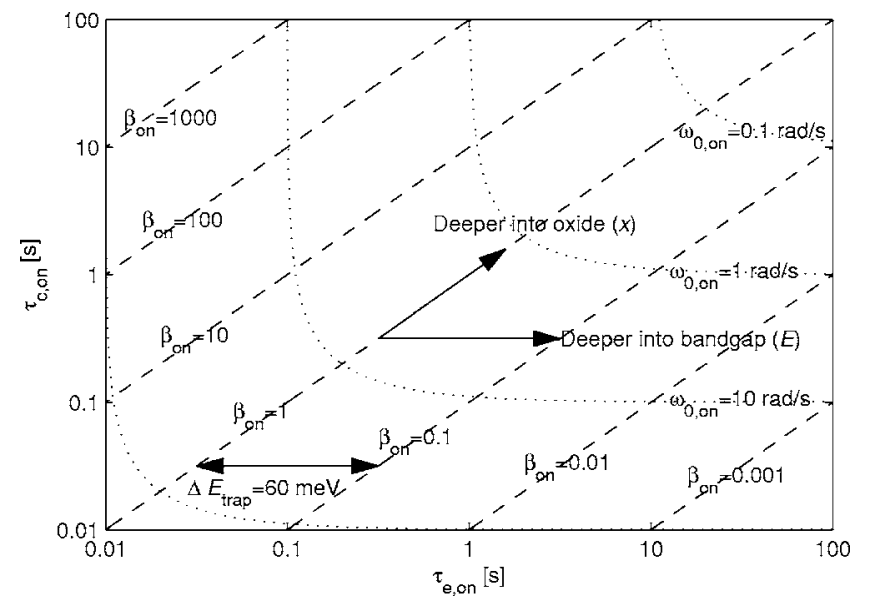

FIG. 2. Parameters of RTS as a function of $\tau_{e, \text { on }}$ and $\tau_{c, \text { on }}$.

$$
\begin{aligned}
\tau_{e, \mathrm{eff}}= & 1 /\left[\frac{d c}{\tau_{e, \mathrm{on}}}+\frac{1-d c}{\tau_{e, \mathrm{off}}}\right] \\
& \times \frac{\tau_{c, \mathrm{eff}}}{1 /\left[\frac{d c}{\tau_{c, \mathrm{on}}}+\frac{1-d c}{\tau_{c, \mathrm{off}}}\right]} .
\end{aligned}
$$

In the limit for $d c \rightarrow 1, \tau_{e, \text { eff }}$ must obviously equal $\tau_{e, \text { on }}$, which is only satisfied in Eq. (7) if

$$
\tau_{c, \text { eff }}=\left[\frac{d c}{\tau_{c, \text { on }}}+\frac{1-d c}{\tau_{c, \text { off }}}\right]^{-1} \text { and } \tau_{e, \text { fff }}=\left[\frac{d c}{\tau_{e, \text { on }}}+\frac{1-d c}{\tau_{e, \text { off }}}\right]^{-1} \text {. }
$$

In summary, a cyclostationary RTS with a constant amplitude and two states, an on state from $t=0 \ldots T d c$, (time constants $\tau_{e, \text { on }}$ and $\left.\tau_{c, \text { on }}\right)$, and an off state from $t=T d c \ldots T$ (time constants $\tau_{e, \text { off }}$ and $\tau_{c \text {,off }}$ ), can, if the switching frequency is sufficiently large, be described by an equivalent stationary RTS with parameters $\tau_{e \text {,eff }}$ and $\tau_{c \text {,eff }}$, expressions for which are given above.

We now investigate how this influences MOSFET RTS noise and relate it to the trap distribution. The power spectral density (PSD) of a unit amplitude RTS is: ${ }^{17}$

$$
S_{\mathrm{RTS}}(\omega)=2 \frac{\beta}{(1+\beta)^{2}} \frac{1}{\omega_{0 \mathrm{RTS}}} \frac{1}{1+\frac{\omega^{2}}{\omega_{0 \mathrm{RTS}}^{2}}}
$$

with

$$
\beta=\frac{\tau_{c}}{\tau_{e}} \text { and } \omega_{0 \mathrm{RTS}}=\frac{1}{\tau_{e}}+\frac{1}{\tau_{c}} .
$$

This also holds for the effective stationary RTS, the time constants of which were derived above (and verified experimentally in Ref. 15). For simplicity, we treat the case for $d c=0.5$.

In Fig. 2, the RTS parameters $\beta_{\text {on }}$ and $\omega_{0, \text { on }}$ are plotted as a function of $\tau_{e, \text { on }}$ and $\tau_{c, \text { on }} . \tau_{e, \text { on }}$ and $\tau_{c, \text { on }}$ are plotted logarithmically along the $x$ and $y$ axes, and contour lines for each factor of 10 difference in $\beta_{\text {on }}$ and $\omega_{0, \text { on }}$ are plotted. A decade change in $\tau_{e}$ at constant $\tau_{c}$ corresponds to a change in trap energy of approximately $60 \mathrm{meV}$. To illustrate which RTS are the dominant contributors to LF noise at the output, the PSD of the RTS at a particular frequency (in this example, 


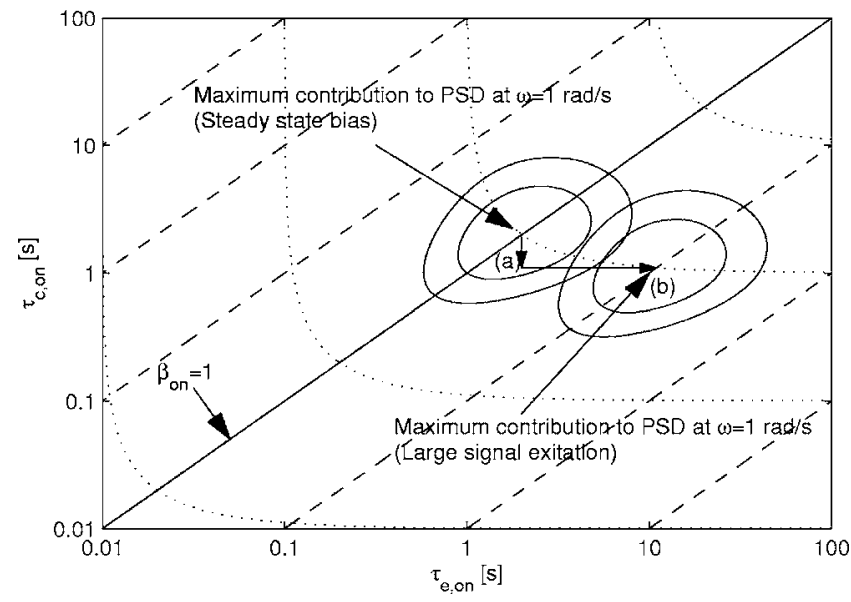

FIG. 3. Contribution to PSD at $\omega=1 \mathrm{rad} / \mathrm{s}$ for different RTSs.

$\omega=1 \mathrm{rad} / \mathrm{s}$ ) is plotted in Fig. 3 as a function of $\tau_{e, \text { on }}$ and $\tau_{c \text {,on. }}$. Contour lines denote the $\tau$ 's of traps with a relative noise power contribution of 80 and $60 \%$ compared to the dominant RTS. The RTSs with $\beta_{\text {on }}$ and $\omega_{0, \text { on }}$ close to 1 give the dominant noise contribution to the PSD at $\omega=1 \mathrm{rad} / \mathrm{s}$. The traps with large or small $\beta_{\text {on }}$ are mostly empty or full, respectively, and do not contribute significantly to the noise. If the RTS corner frequency is too low or too high (compared to $1 \mathrm{rad} / \mathrm{s}$ ), the contribution of the RTS is insignificant as well. $^{18}$

We now examine RTS behavior when the device is periodically turned on and off. For this, bias dependence of $\tau_{e}$ and $\tau_{c}$ is modeled in a very simple and insightful way: $\tau_{e, \text { off }}=\tau_{e, \text { on }} / m_{e}$ and $\tau_{c, \text { off }}=\tau_{c, \text { on }} \cdot m_{c}$, respectively. From this and from Eq. (8), it follows that $\tau_{e, \text { eff }}=\left(2 /\left(m_{e}+1\right)\right) \tau_{e, \text { on }}$ and $\tau_{c \text {,eff }}=\left(2 m_{c} /\left(m_{c}+1\right)\right) \tau_{c, \text { on }}$. This changes the effective time constants of all RTSs in the device. In Fig. 3, it is shown for $m_{e}=m_{c}=10$ that [Fig. 3(a)] $\tau_{c}$ has decreased by a factor of 1.8 and [Fig. 3(b)] that $\tau_{e}$ has increased by a factor of 5.5 compared to the steady-state situation. The main contribution to the PSD is therefore now from different RTSs. Traps that were mostly full in the steady-state case now have an occupancy closer to $50 \%$, and they dominate output noise.

The shape of the noise contribution curve in Fig. 3 does not change; it has only been shifted along the $\log \tau_{e}$ and $\log \tau_{c}$ axes. Hence, the noise PSD will not change at all if the distribution of $\tau \mathrm{s}$ is uniform in $\log t$. Such a uniform distribution in $\log t$ results if two conditions are satisfied. First, the distribution of trap depth in the oxide, $x$, should be uniform. (This is the basis of McWhorter's model ${ }^{19}$ and responsible for the emergence of a $1 / f$ spectrum. $)^{20}$ Second, if the trap energy level $E$ is uniformly distributed throughout the band gap, a uniform distribution of traps in $\log \tau_{e}$ and $\log \tau_{c}$ results.

However, in measurements, it is often seen that turning a device on and off periodically leads to a decrease in the LF noise PSD. ${ }^{4,5,8,9}$ Logically, then, the distribution of traps is not uniform in $\log t$. (This can be the distribution of $\tau \mathrm{s}$ in a large device with very many traps, but it can also be the distribution of $\tau \mathrm{s}$ over an ensemble of small devices, each with a limited number of traps. $)^{21}$ One likely scenario ${ }^{10}$ is that the trap density near the center of the band gap is lower. ${ }^{22}$ Figures 2 and 3 show that traps closer to the center of the band gap are the ones contributing LF noise when the device is periodically turned on and off, and if there are fewer traps, this explains why turning the device on and off lowers the LF noise.

In conclusion, we have shown that when a MOSFET is periodically and rapidly ${ }^{23}$ turned on and off, its time-variant RTS (with time constants $\tau_{e \text {,on }}, \tau_{c \text {,on }}, \tau_{e \text {, off }}$, and $\tau_{e \text {,on }}$ ) can be modeled by an equivalent stationary RTS with time constants $\tau_{e \text {,eff }}$ and $\tau_{c \text {,eff }}$. Using Shockley-Read-Hall statistics applied under transient conditions, we have derived expressions for these equivalent RTS time constants. Making use of the concept of an equivalent time constant, we demonstrate that under these conditions, which are similar to what MOSFETs encounter in many circuits, traps closer to the center of the band gap dominate the LF noise performance of the device. This links two important previous observations, namely on the one hand that trap densities in MOSFETs are commonly U-shaped in energy, ${ }^{10}$ and on the other hand that LF noise in MOSFETs decreases when the device is periodically turned on and off. $4,5,8,9$

${ }^{1}$ P. H. Woerlee, M. J. Knitel, R. van Langevelde, D. B. M. Klaassen, L. F. Tiemeijer, A. J. Scholten, and A. T. A. Zegers-van Duijnhoven, IEEE Trans. Electron Devices 48, 1776 (2001).

${ }^{2}$ M. J. Kirton and M. J. Uren, Adv. Phys. 38, 367 (1989).

${ }^{3}$ T. G. M. Kleinpenning, Physica B 164, 331 (1990).

${ }^{4}$ I. Bloom and Y. Nemirovsky, Appl. Phys. Lett. 58, 1664 (1991).

${ }^{5}$ B. Dierickx and E. Simoen, J. Appl. Phys. 71, 2028 (1992).

${ }^{6}$ P. Larsson, IEEE J. Solid-State Circuits 34, 1951 (1999).

${ }^{7}$ S. Ye, L. Jansson, and I. Galton, in Proc. CICC (IEEE, New York, 2003), pp. 13.2.1-13.2.4.

${ }^{8}$ A. P. van der Wel, E. A. M. Klumperink, S. L. J. Gierkink, R. F. Wassenaar, and H. Wallinga, IEEE Electron Device Lett. 21, 43 (2000).

${ }^{9}$ A. P. van der Wel, E. A. M. Klumperink, and B. Nauta, Electron. Lett. 37, 55 (2001).

${ }^{10}$ X. L. Xu, R. T. Kuhn, J. J. Wortman, and M. C. Öztürk, Appl. Phys. Lett. 60, 6063 (1992)

${ }^{11}$ M. Schulz and H. H. Mueller, Adv. Solid State Phys. 35, 229 (1996).

${ }^{12}$ W. Shockley and W. T. Read, Jr., Phys. Rev. 87, 835 (1952).

${ }^{13}$ N. B. Lukyanchikova, M. V. Petrichuk, and N. P. Garbar, in Proc. 14th ICNF (World Scientific, Leuven, 1997), pp. 232-235.

${ }^{14}$ Z. Shi, J. Miéville, and M. Dutoit, IEEE Trans. Electron Devices 41, 1161 (1994).

${ }^{15}$ J. S. Kolhatkar, E. Hoekstra, C. Salm, A. P. van der Wel, E. A. M. Klumperink, J. Schmitz, and H. Wallinga, in Proc. IEDM (2004).

${ }^{16}$ A. P. van der Wel, Ph.D. thesis, University of Twente, (2005).

${ }^{17}$ S. Machlup, J. Appl. Phys. 25, 341 (1954).

${ }^{18}$ In general, the dominant contribution to output noise always comes from traps with $\beta$ close to 1 and $\omega_{0 \mathrm{RTS}}$ close to the frequency of interest.

${ }^{19}$ A. L. McWhorter, Ph.D. thesis, MIT, Cambridge, MA (1955).

${ }^{20}$ Direct tunnelling is assumed, making the capture cross section a negative exponential function of $x$.

${ }^{21}$ An individual small device will behave according to the traps it happens to contain, but the ensemble average of the noise performance will be the same as for a single large device with many traps, assuming that individual traps make uncorrelated contributions to the output noise.

${ }^{22}$ The trap depth distribution in the oxide may still be uniform: Nonuniformity in $E$ does not preclude the emergence of a $1 / f$ spectrum.

${ }^{23}$ Much faster than $\tau_{e}$ and $\tau_{c}$. 